\title{
Resistive Plate Chambers in the ARGO-YBJ experiment: operational features, monitoring and control
}

\author{
P. Camarri*i \\ University of Roma "Tor Vergata" and INFN Roma Tor Vergata \\ E-mail: paolo.camarrieroma2.infn.it
}

\begin{abstract}
The ARGO-YBJ experiment has been running almost uninterruptedly with its complete layout since October 2007. The stable behaviour of the detector, a full-coverage array of Resistive Plate Chambers (RPCs) covering a surface of $74 \times 78 \mathrm{~m}^{2}$ surrounded by a guard ring, allowed this. Nevertheless, the work to optimize the stability of the experimental setup is still going on. The monitoring information provided by the Detector Control System (DCS) is crucial in this respect. The recorded daily and seasonal changes of temperature and barometric pressure affect the density of the gas mixture inside the detector and consequently its behaviour, in particular the time resolution and, to a lesser degree, the efficiency. The aim of this study is to develop a straightforward procedure in order to equalize and possibly stabilize the detector response.
\end{abstract}

XI workshop on Resistive Plate Chambers and Related Detectors - RCP2012,

February 5-10, 2012

INFN Laboratori Nazionali di Frascati Italy

\footnotetext{
* Speaker.

† on behalf of the ARGO-YBJ Collaboration.
} 


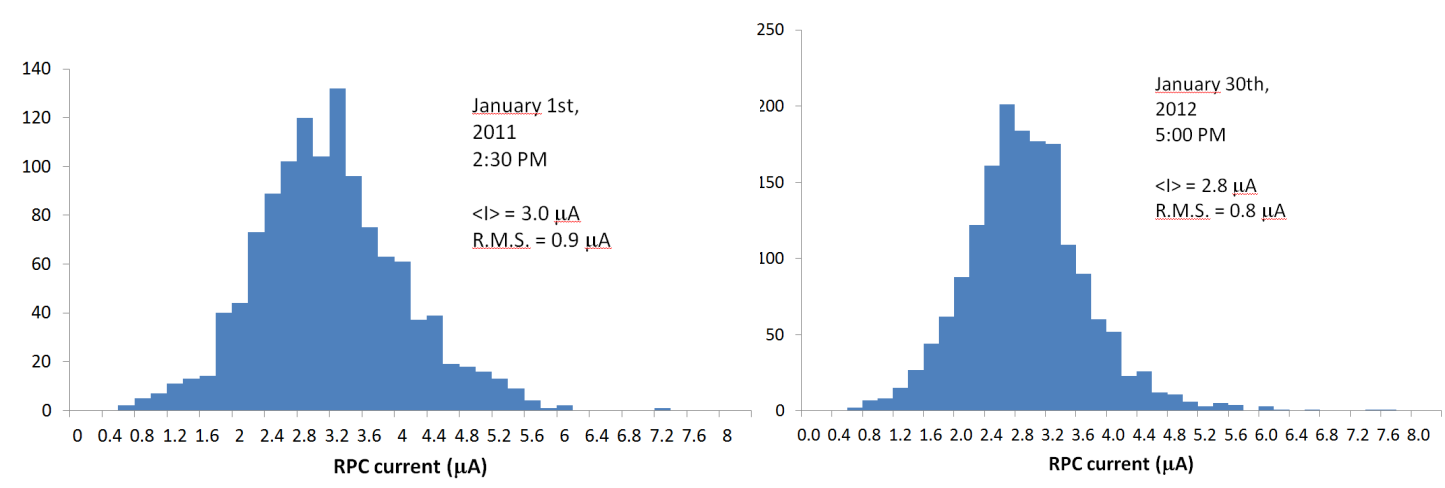

Figure 1: Current distributions of the ARGO-YBJ RPCs recorded on Jan 1, 2011 (left) and Jan 30th, 2012 (right).

\section{Stability of the ARGO-YBJ RPCs}

The stability of the detector response is mandatory in the ARGO-YBJ experiment, which is supposed to run uninterruptedly for at least 5 years. The main features of ARGO-YBJ and its physics performance were discussed in another contribution to this workshop [W]. The present discussion is focused on the stability of the current absorbed by the detector and on the development of a procedure to stabilize the detector response.

The current absorbed by the RPCs in ARGO-YBJ [[] looks pretty stable as shown in Fig. 四, showing the current distributions on Jan 1st, 2011 and Jan 30th, 2012. The mean values and R.M.S. are practically the same. The population in the more recent plot is greater due to the addition of a substantial number of chambers in the monitoring chain after a maintenance operation.

\section{Operating voltage of the ARGO-YBJ RPCs}

As reported in previous works, the gas gain in RPCs depends on the environmental conditions, namely on the local temperature and barometric pressure [B]]. If $V_{\text {app }}$ is the fixed applied voltage when the absolute temperature is $T$ and the barometric pressure is $p$, then the effective voltage, if the reference absolute temperature and barometric pressure are chosen to be $T_{0}$ and $p_{0}$ respectively, is

$$
V_{e f f}=V_{a p p} \frac{T}{T_{0}} \frac{p_{0}}{p}
$$

Standard operating conditions for the ARGO-YBJ RPCs correspond to applied voltage $V_{0}$ at absolute temperature $T_{0}$ and barometric pressure $p_{0}$. Accounting for the fact that the effect of temperature changes affects the ARGO-YBJ RPCs with a delay of about one hour [䧃], the operating-point regulation rule is:

$$
V_{\text {app }}(t)=V_{0} \frac{T_{0}}{p_{0}} \frac{p(t)}{T(t-1 \mathrm{~h})}
$$

This algorithm is meant to stabilize the gas gain inside the RPCs, so that the detector can always work at constant operating conditions. 


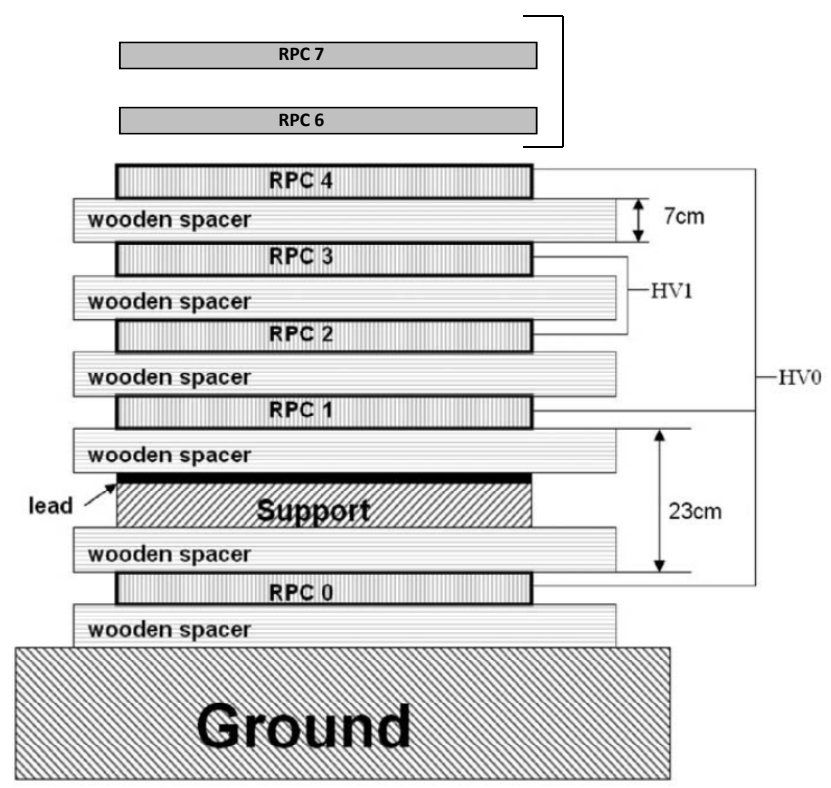

Figure 2: Layout of the cosmic-ray telescope located by the ARGO-YBJ RPC carpet.

\section{Testing and monitoring facility at the ARGO-YBJ site}

A small-size cosmic-ray telescope was installed close to the South side of the ARGO-YBJ carpet as a monitoring and testing facility for the RPCs. It has been already used to study the dependence of the detector efficiency and time resolution on the temperature [\$]. For the present study [ [6] the cosmic-ray telescope has been slightly modified, and its basic layout is shown in Fig. [2.

This telescope is connected to the general ARGO-YBJ acquisition system, so its data are recorded according to the standard trigger condition on the ARGO-YBJ carpet. Therefore, the analysis on the telescope must be performed with an off-line procedure. Cosmic-ray tracks are triggered by a coincidence of the chambers 0,1 and 4 . These three chambers, together with the chambers 2 and 3, are powered at $7200 \mathrm{~V}$ which is the standard voltage applied to the RPCs in the ARGO-YBJ carpet. The telescope has been running since 2008, using the chambers 2 and 3 as the test chambers.

\section{Extension of the test with the monitor telescope}

With respect to the initial telescope layout, chambers 6 and 7 were added to the stack. These two chambers were powered according to the algorithm described previously in Eq. 2.2. This way, a continuous comparison could be made between a "standard" behaviour in which the applied voltage is left unchanged and a "corrected" behaviour in which the applied voltage is suitably regulated to compensate the gas-gain changes. It is necessary to point out that the trigger configuration of the telescope, as described previously, is not optimized for the geometrical acceptance of the two 


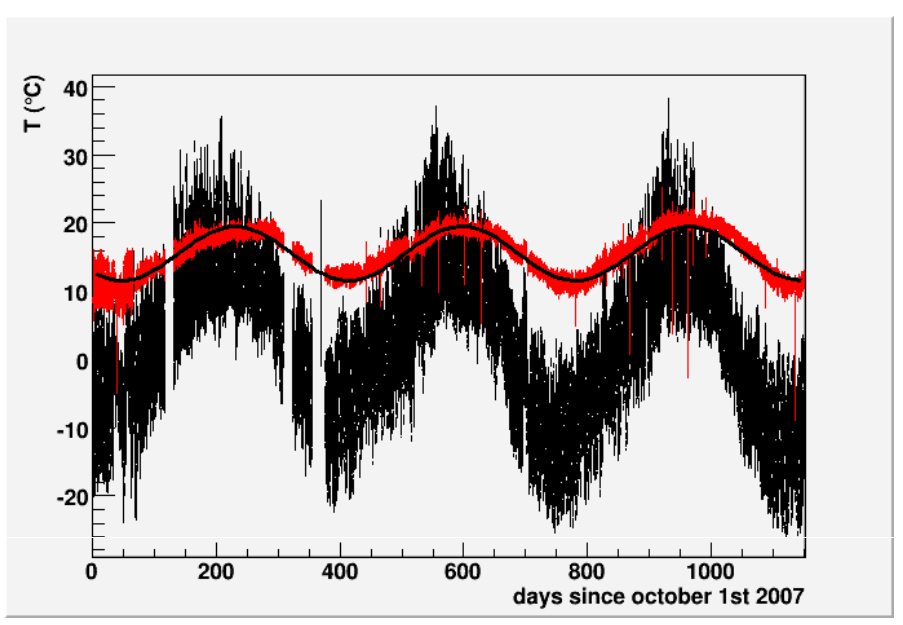

Figure 3: Temperature at the ARGO-YBJ experimental site, both outside and inside (dark and light points respectively), since the start of the data taking in October, 2007. The long-term regularity of the inner temperature is pointed out with a sinusoidal fit of the experimental points: the yearly average value is 15.5 ${ }^{\circ} \mathrm{C}$.

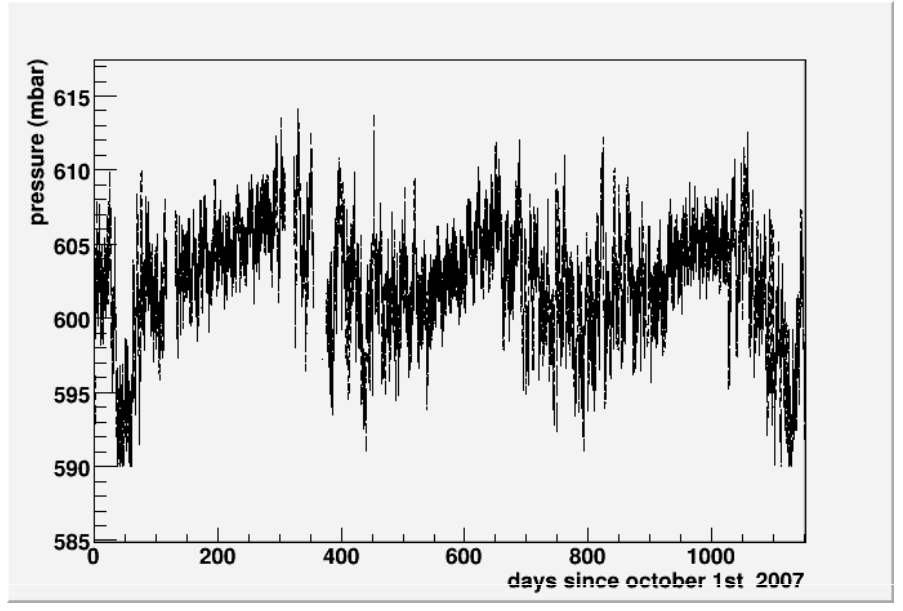

Figure 4: Time trend for the barometric pressure since the start in October, 2007. The yearly average value is about 600 mbar.

added chambers. Therefore every efficiency measurement performed on chambers 6 and 7 will actually give a value of the efficiency times the geometrical acceptance. However, this is not a crucial issue in this test. The reference values for the absolute temperature and the barometric pressure are chosen to be close to the yearly average values provided by the monitoring system (DCS), namely $T_{0}=288.65 \mathrm{~K}$ and $p_{0}=600$ mbar.

Figure B] shows the time evolution of the temperature at the ARGO-YBJ experimental site over 3 years. In particular, the temperature inside the experimental hall shows a 1-year period with average value equal to $15.5 \mathrm{C}$. Figure $⿴ 囗 十$ shows the time evolution of the barometric pressure at the ARGO-YBJ experimental site over the same time span. It shows a 1-year period with average value 


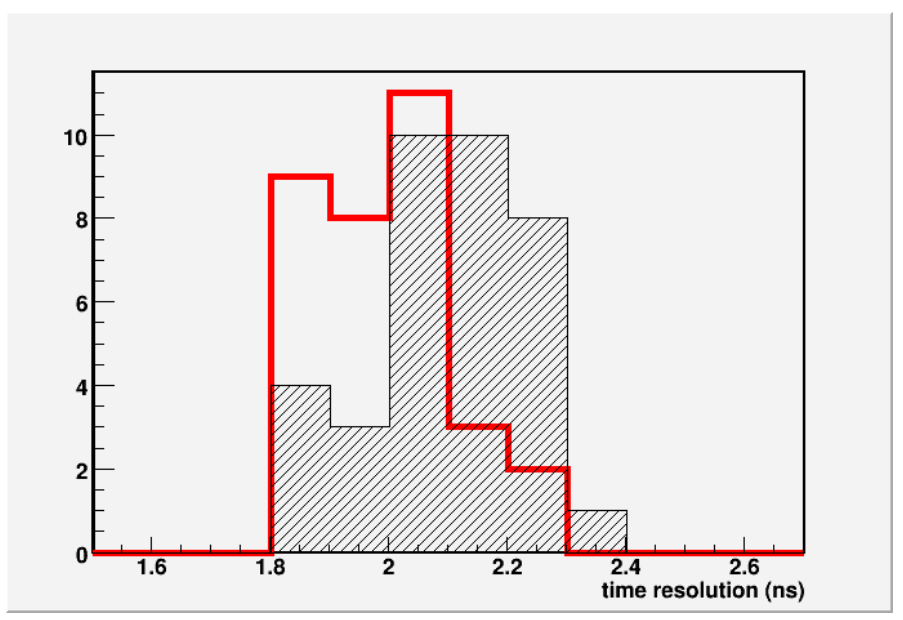

Figure 5: Distribution of the RPC time resolution as obtained from the measurement of the time of flight between chambers 6 and 7. The filled distribution refers to January 9-12, 2011, with a r.m.s. of 133 ps. The superimposed distribution with no filling refers to January 12-15, 2011, with a r.m.s. of 98 ps.

about 600 mbar. The DCS, which includes all the monitoring and control procedures of the ARGOYBJ experiment, must act on RPCs 6 and 7 at regular time intervals so that the voltage change to be applied each time is not greater than a few Volts. In this test, the time interval was chosen to be 15 minutes. The complete high-voltage control algorithm also includes safety checks: for instance, the "new" voltage to be applied is used only if it is close enough to the previous applied voltage, to avoid critical mistakes induced by possible failures in the readout of the environmental sensors. In addition, limits on the current absorbed by the power supply are set, so that the detectors are protected against any possible dangerous increase of current.

\section{Implementation of the high-voltage control on the test facility}

The voltage-control procedure on the chambers 6, 7 in the cosmic-ray telescope started on January 12th, 2011. The behaviour of these two chambers immediately before and immediately after this was compared.

Figure 5 shows the histograms of the time resolution obtained from the measurement of the time of flight between chambers 6 and 7, for the period January 9-12, 2011 (shaded) and for the period January 12-15, 2011. The voltage-control procedure led to a slight change in the r.m.s. of the distribution, which decreased from 133 ps to 98 ps.

Figure 6 shows the histograms of the average efficiency times the geometrical acceptance for chambers 6 and 7, for the period January 9-12, 2011 (upper, black) and for the period January 1215, 2011 (lower, red). The use of voltage-control procedure decreased the r.m.s. of the distribution from $1.1 \%$ to $0.5 \%$.

On January 31st, 2012 the role of the tested RPC pairs in the telescope was exchanged: RPCs 6 and 7 were set at fixed applied voltage $(7200 \mathrm{~V})$ and RPCs 2 and 3 were put under regulated applied voltage. 


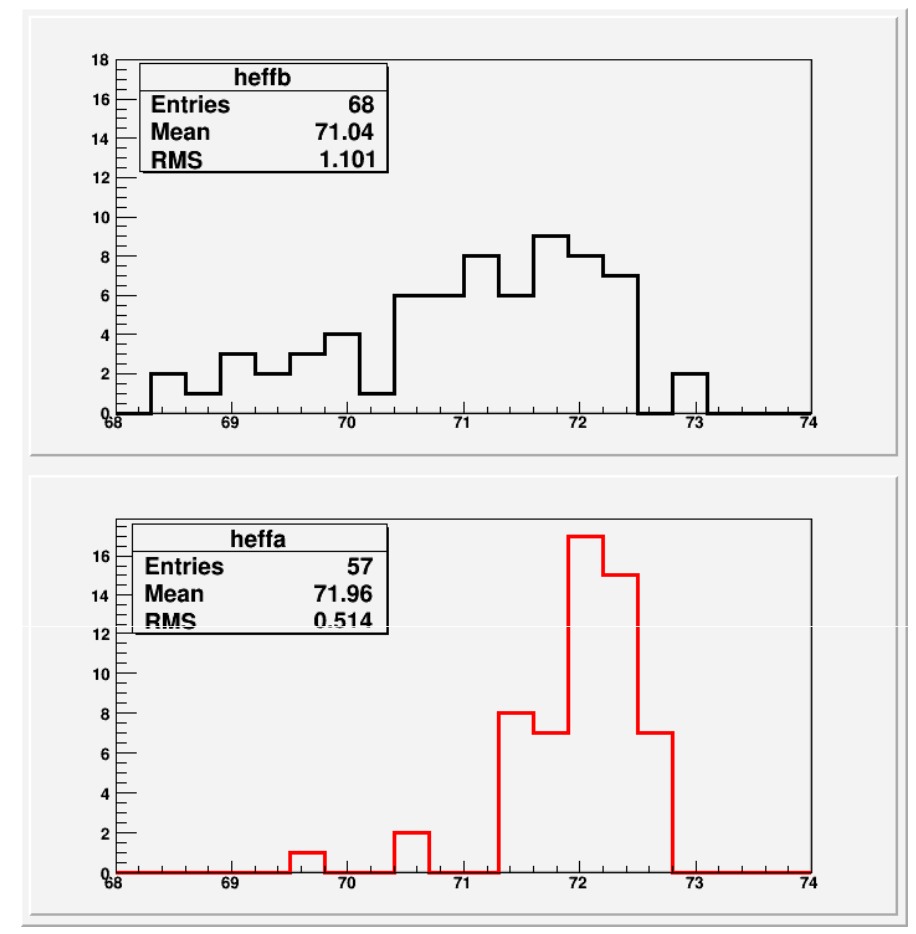

Figure 6: Distribution of the RPC efficiency times acceptance (in \%) for chambers 6 and 7 . The upper distribution refers to January $9-12,2011$, with a r.m.s. of $1.1 \%$. The lower distribution refers to January $12-15,2011$, with a r.m.s. of $0.5 \%$.
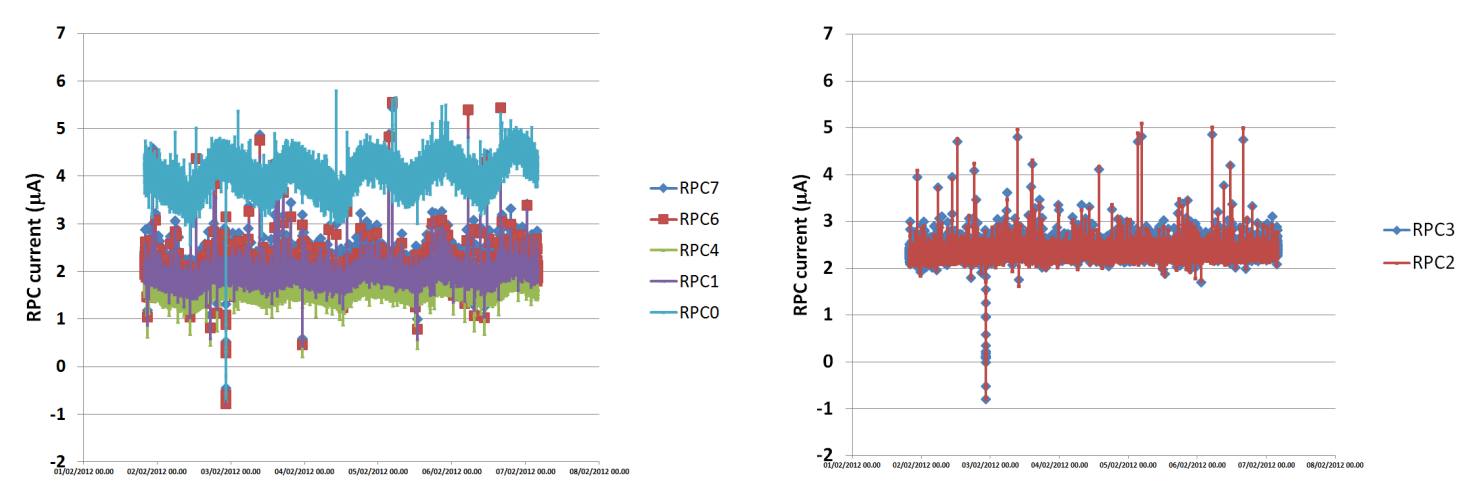

Figure 7: Time trends (from February 2 till 7, 2012) for the currents of the test RPCs with fixed applied voltage (left) and regulated applied voltage (right). 

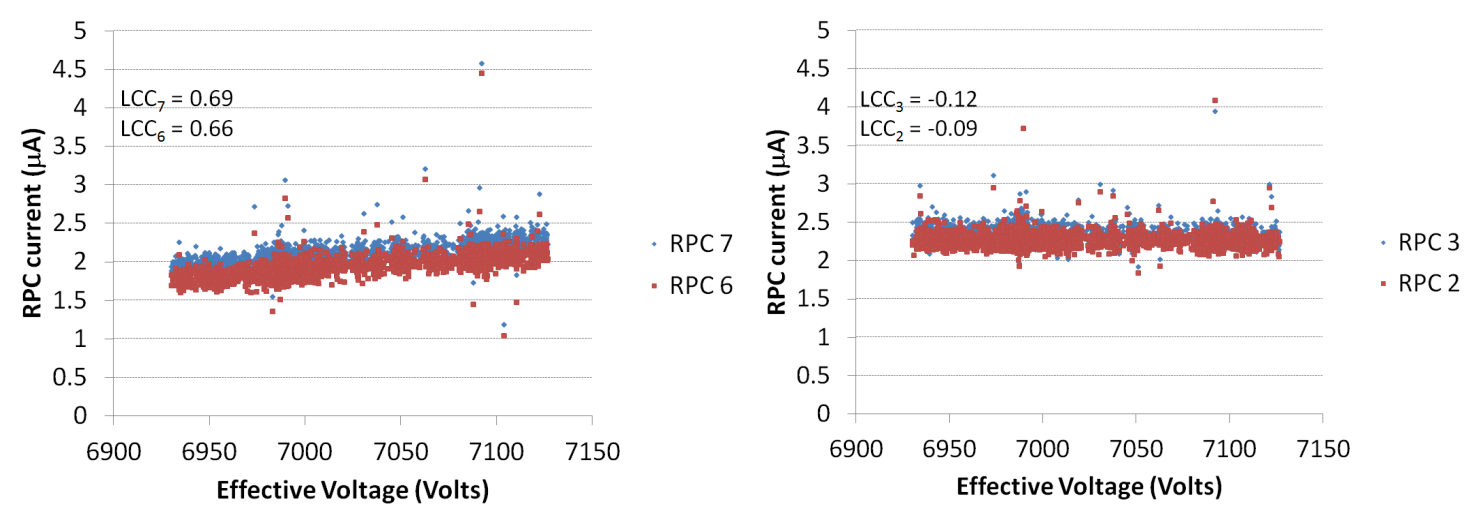

Figure 8: Current vs effective voltage correlation plots for the test RPCs with fixed applied voltage (left) and regulated applied voltage (right).

Fig. $\square$ shows a 5-day time trend for the five chambers with fixed voltage and for the two chambers with voltage regulation. The use of the regulation algorithm clearly makes the current very stable.

Fig. 8 shows the current vs effective voltage correlation plots for the test pair with fixed applied voltage and for the test pair with regulated applied voltage. The respective values for the linear correlation coefficients give a further confirmation that the voltage-feedback algorithm is in agreement with the expectations.

\section{Conclusions}

This test leads to the following conclusions:

- Before starting the voltage-control procedure, the efficiency times the geometrical acceptance of the chambers 6 and 7 varied between $67 \%$ and $73 \%$ with a r.m.s. greater than $1 \%$. The time resolution obtained from the measurement of the time of flight between the RPCs 6 and 7 varied between $1.8 \mathrm{~ns}$ and $2.5 \mathrm{~ns}$ with a r.m.s. of 133 ps.

- After applying the voltage-control procedure, the efficiency times the geometrical acceptance of the chambers 6 and 7 varies between $70 \%$ and $72.5 \%$ with a r.m.s. about $0.5 \%$. The time resolution obtained from the measurement of the time of flight between the RPCs 67 varies between $1.8 \mathrm{~ns}$ and $2.2 \mathrm{~ns}$ with a r.m.s. of $98 \mathrm{ps}$.

- The voltage-control procedure makes the absorbed current of the test RPCs very stable.

The voltage regulation under study acts only on the RPC absorbed current and intrinsic time resolution. The effect of the voltage-control procedure is visible in spite of the reduced spread of the operating parameters (efficiency times geometrical acceptance, and time resolution): the main result obtained in this test is an extreme stabilization of the operating current of the RPCs. The more regular working conditions for RPCs resulting from this are crucial in view of the time extension 
of experiments like ARGO-YBJ which will have to keep running uninterruptedly and reliably for several years.

\section{References}

[1] R. Iuppa on behalf of the ARGO-YBJ Collaboration; contribution to this workshop.

[2] G. Aielli et al.; NIM A 562 (2006) 92-96.

[3] P. Camarri on behalf of the ARGO-YBJ Collaboration; Proceedings of the 29th ICRC, Pune (India), Aug 3-10, 2005. P. Camarri on behalf of the ARGO-YBJ Collaboration; Proceedings of the 30th ICRC, Merida (Mexico), Jul 3-11, 2007.

[4] P. Camarri, C.Y. Wu on behalf of the ARGO-YBJ Collaboration; Proceedings of the 31st ICRC, Łódź (Poland), Jul 7-15, 2009.

[5] G. Aielli et al.; NIM A 608 (2009) 246-250.

[6] P. Camarri on behalf of the ARGO-YBJ Collaboration; Proceedings of the 32nd ICRC, Beijing (China), Aug 11-18, 2011. 